\title{
Near-Edge X-Ray Absorption Fine-Structure Investigation of Graphene
}

\author{
D. Pacilé, ${ }^{1, *}$ M. Papagno, ${ }^{1}$ A. Fraile Rodríguez, ${ }^{2}$ M. Grioni, ${ }^{3}$ and L. Papagno ${ }^{1}$ \\ ${ }^{1}$ Istituto Nazionale di Fisica Nucleare (INFN) and Dipartimento di Fisica Università della Calabria, \\ 87036 Arcavacata di Rende, Cosenza, Italy \\ ${ }^{2}$ Swiss Light Source, Paul Scherrer Institut, 5232 Villigen PSI, Switzerland \\ ${ }^{3}$ Institut de Physique des Nanostructures, Ecole Polytechnique Fédérale de Lausanne (EPFL), CH-1015 Lausanne, Switzerland
}

Ç.Ö. Girit, J. C. Meyer, G. E. Begtrup, and A. Zettl

Department of Physics, University of California, Berkeley, California 94720, USA

Materials Sciences Division, Lawrence Berkeley National Laboratory, Berkeley, California 94720, USA

(Received 16 May 2008; published 8 August 2008)

\begin{abstract}
We report the near-edge $x$-ray absorption fine-structure (NEXAFS) spectrum of a single layer of graphite (graphene) obtained by micromechanical cleavage of highly ordered pyrolytic graphite on $\mathrm{SiO}_{2}$ substrate. We utilized a photoemission electron microscope to separately study single-, double-, and fewlayers graphene samples. In single-layer graphene we observe a splitting of the $\pi^{*}$ resonance and a clear signature of the predicted interlayer state. The NEXAFS data illustrate the rapid evolution of the electronic structure with the increased number of layers.
\end{abstract}

PACS numbers: 73.20.At, 73.90.+f

The recent discovery of a single sheet of graphite [1], called graphene, has opened up a new area of condensed matter physics. Graphene proves that materials just one atom thick may exist, with exciting prospects for applications. Its unusual electronic spectrum, where charge carriers mimic massless relativistic particles [2,3], also provides an unexpected bridge between condensed matter physics and quantum electrodynamics.

The method to obtain single sheets of graphite [1], called micromechanical cleavage, allows easy production of sample with a typical size of few tens of microns, ideal for ballistic transport and quantum Hall effect measurements, but inappropriate for many conventional spectroscopy investigations in ultrahigh vacuum (UHV) conditions. In the absence of new and more efficient ways to make graphene, samples obtained by micromechanical cleavage of bulk graphite are used in a limited class of experiments, where the size and the identification of thin flakes is possible. Indeed, after the cleavage with simple adhesive tape, graphene crystallites left on the $\mathrm{SiO}_{2}$ substrate are extremely rare and hidden among hundreds of thicker flakes. Conventional surface science probes of the electronic and structural properties of materials, are then ruled out, unless they are coupled to a microscope. On the other hand, single- and few-layer graphene (FLG) samples have been grown epitaxially by chemical vapor deposition of hydrocarbons on metal substrates [4] and by thermal decomposition of $\mathrm{SiC}$ [5]. In both cases, the hybridization of graphene with the substrate is an unavoidable complication, although graphene on $\mathrm{SiC}$ preserves most of the electronic properties expected for a free layer $[6,7]$.

In this Letter we report the near-edge $\mathrm{x}$-ray absorption fine structure (NEXAFS) spectra of a free layer of graphene, and of FLG samples, obtained by a photoemission electron microscope (PEEM) in UHV conditions. The spectrum of graphene exhibits a new structure below the $\pi^{*}$ resonance, reflecting its peculiar density of states (DOS) above the Fermi level [8], and a peak between the $\pi^{*}$ and $\sigma^{*}$ resonances. We attribute the latter to the analog of the interlayer state of graphite, which was predicted to exist even in single-layer graphene $[9,10]$.

Our samples were prepared by micromechanical cleavage of HOPG on $\mathrm{SiO}_{2}$ substrates and characterized by optical microscopy (OM) and Raman spectroscopy, to identify single layers of graphite and thicker flakes $[11,12]$. The laterally resolved NEXAFS experiments were carried out at the surface-interface microscopy (SIM) beam line [13] of the Swiss Light Source, using an Elmitec photoemission electron microscope (PEEM) equipped with an energy analyzer. To obtain elementspecific PEEM contrast, images collected at the peak of the $\mathrm{C} K$ edge $(285.5 \mathrm{eV})$ were normalized by corresponding images measured below the edge $(282.5 \mathrm{eV})$. Figure 1 shows a direct comparison between OM images [(a), (c), and (e)] and PEEM ones [(b), (d), and (f)] of some selected samples (S1, S2, and S3). Sample S3 was prepared using a different technique: trenches $2 \mu \mathrm{m}$ wide were patterned on the $\mathrm{SiO}_{2} / \mathrm{Si}$ substrate by photolithography and then etched to a depth of $150 \mathrm{~nm}$. After the cleavage procedure, single sheets suspended over trenches provided free-standing graphene membranes. PEEM images were collected by setting the photon energy just above the $\mathrm{C} K$ edge, for a total exposure time of about $30 \mathrm{~s}$. The contrast provided by the PEEM is surprisingly high, even for a single layer, demonstrating the possibility to identify and analyze the electronic properties of graphene in a UHV experiment. Moreover, different thickness of the flakes, quantified by means of the OM contrast method [11], can be easily recognized in the PEEM images. 


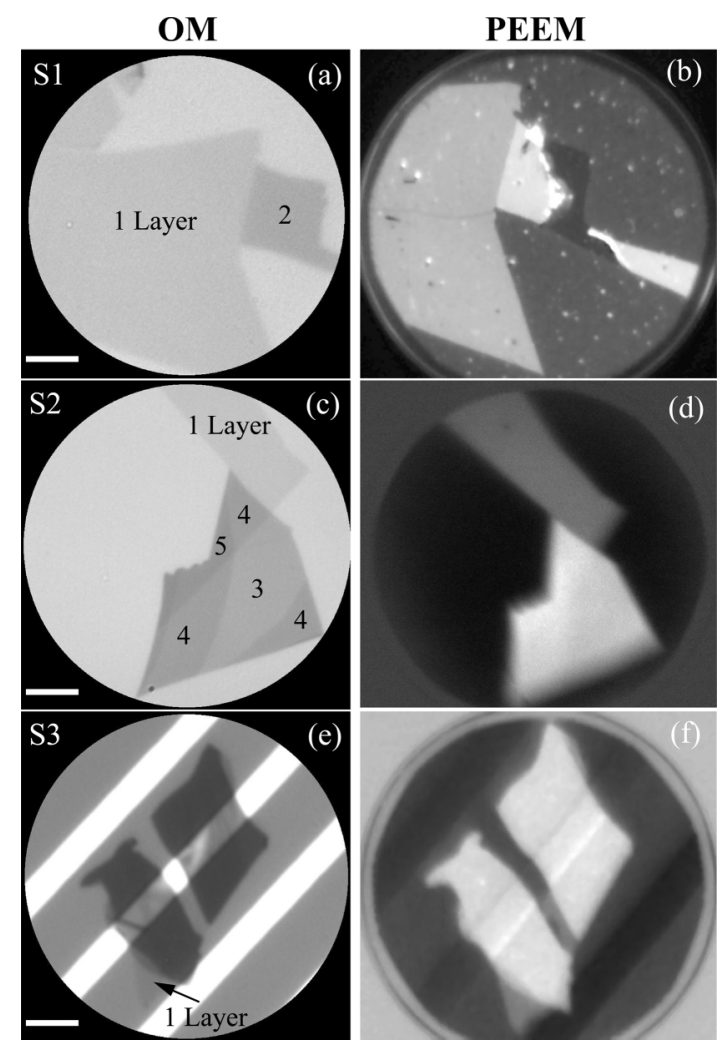

FIG. 1. OM images [(a), (c), and (e)] and PEEM ones [(b), (d), and (f)] of some selected samples (S1, S2, and S3). Scale bars are: $5 \mu \mathrm{m}$ in (a) and (e); $10 \mu \mathrm{m}$ in (c).

Photoabsorption spectra were extracted by processing a full package of PEEM images obtained by tuning the photon energy across the $\mathrm{C} K$ edge, and recording the total electron yield (TEY) from the region of interest. To normalize the C $K$ absorption spectra, the NEXAFS scans were divided by a scan taken from an area of the substrate. All measurements were performed at grazing incidence $\left(16^{\circ}\right)$ with a linear polarization almost perpendicular to the basal plane of graphite, a configuration $(\mathbf{E} \| \mathbf{c})$ which enhances transitions into final states of $\pi$ symmetry [14]. Figure 2 shows $C K$ absorption spectra obtained for graphene, bilayer graphene, and FLG samples. The main peak at $285.5 \mathrm{eV}$ ( $\pi^{*}$ resonance $)$, is associated with the conduction $\pi$ states around the $M$ and $L$ points of the Brillouin zone (BZ) [10]. The structure at $291.5 \mathrm{eV}$ is due to the dispersionless $\sigma$ states at the $\Gamma$ point of the BZ. Higher energy features are due to transitions towards higher-lying states of $\pi$ or $\sigma$ symmetry [14]. They are better defined in thicker samples (above 5 layers), where the electronic structure is mainly reminiscent of the graphite band structure. The electronic structure of graphite exhibits strong modifications for a finite number of layers, although the interlayer coupling between its planes is supposed to be very weak. Graphene is a zero-gap semiconductor with a linear Dirac-like spectrum around the Fermi energy $\left(E_{F}\right)$, while graphite is a semimetal with a band overlap of $41 \mathrm{meV}$ [15]. In the intermediate region, FLG show differ-

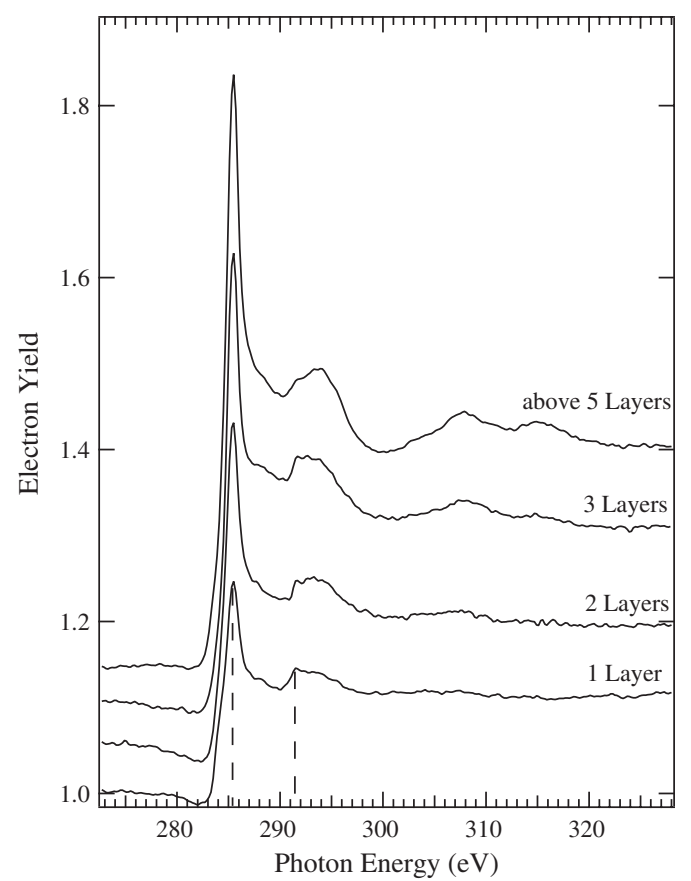

FIG. 2. C $K$-edge photoabsorption spectra of (from the bottom): graphene, bilayer graphene, and FLG samples. The dashed lines show the $\mathrm{C} 1 s \pi^{*}$ and $\mathrm{C} 1 s \sigma^{*}$ transitions.

ent paraboliclike $\pi$ states, with an increasing band overlap as a function of the number of layers, up to that of graphite above ten layers. In a band-theory approach [16], the nearedge structure is the sum of the transition rates to all possible unoccupied one-electron states. Each transition rate is further separated into two components: the matrix element between the initial and the final states, and the DOS projected along the $k_{z}$ direction. Both terms vary with the number of graphene layers. Moreover, matrix elements strongly depend on polarization. In graphite, above the first transition ( $\pi^{*}$ resonance) final states have mainly $\sigma$ symmetry [14]. Their contribution to the NEXAFS spectrum is strongly reduced in our experimental set-up $(\mathbf{E} \| \mathbf{c})$ and this explains why the spectra of Fig. 2 are dominated by the first resonance. The absence of those high energy features is particularly evident in the photoabsorption spectrum of graphene (Figs. 2 and 4), where, in addition, the number of absorbing atoms per unit area is reduced to its minimum value. Near-edge structures can also be interpreted by multiple-scattering theory [17], which considers the scattering of the excited electron wave function by the neighboring atoms. The presence of a cage around the absorbing atom is reflected into the features of the near-edge structure, thus explaining the absence of signal for a single layer (Figs. 2 and 4) when the polarization of the light would select neighboring atoms above and below the plane.

Figure 3 shows a close-up around the $\pi^{*}$ resonance of graphene, bilayer graphene, and FLG spectra extracted from Fig. 2. Here, a clear peak located below the $\pi^{*}$ resonance, at about $283.7 \mathrm{eV}$, can be seen for graphene, a broader shoulder is present for bilayer graphene, while no 


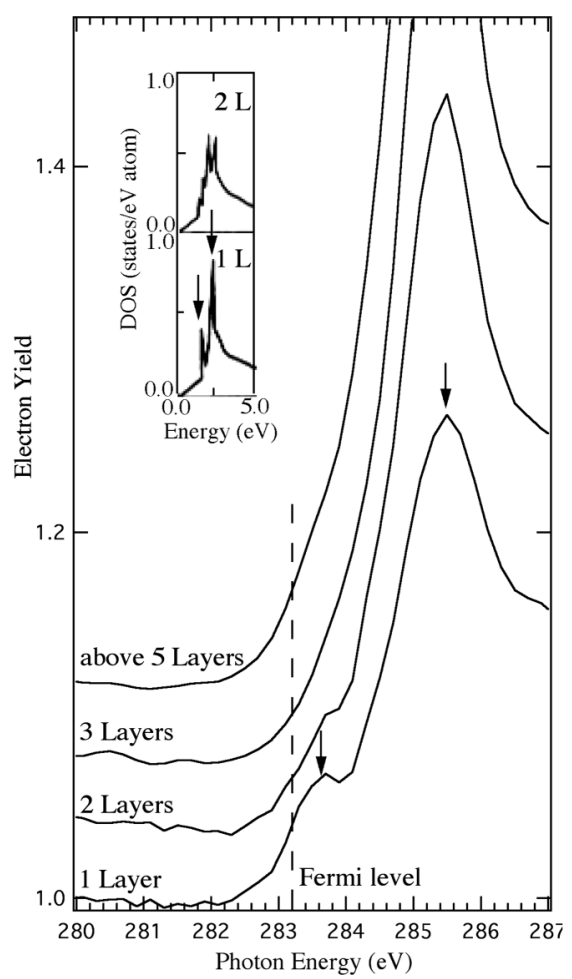

FIG. 3. Expanded C $K$-edge photoabsorption spectra extracted from Fig. 2. The inset shows DOS calculations of single- and double-layer graphene [8], in the energy region (0.0-5.0) eV above $E_{F}$. The Fermi level, as extracted from Ref. [8], is also reported in the graph. Arrows show the double structure of the $\pi^{*}$ resonance in graphene. The separation between the two features in the theoretical DOS of single-layer graphene is about $0.8 \mathrm{eV}$, while the experimental separation is between 1.2 and $1.8 \mathrm{eV}$ for our samples.

clear signature at this energy is present above two layers of graphene. A similar structure was observed in NEXAFS measurements performed on nanographite grains growth on $\mathrm{Pt}(111)$, and explained in term of an edge-derived electronic state [18]. Specifically, it was assigned to grains with zigzag edges, for which edges states are expected just above the Fermi level, in contrast with grains with armchair edges [19]. Our measurements show a similar structure, but the edge contribution is negligible considering the size $(>10 \mu \mathrm{m})$ of our samples. On the other hand, the DOS (inset of Fig. 3) of 1 and 2 layers of graphene, calculated in the local-density approximation by Trickey et al. [8], is in good agreement with the experimental data. Considering the energy resolution of our spectra ( $0.2 \mathrm{eV}$ ), the four peaks (starting $1 \mathrm{eV}$ above the Fermi level) in the DOS of bilayer graphene will yield a shoulder below the $\pi^{*}$ resonance. On the other hand, two pronounced peaks are present in the DOS of graphene, which are clearly resolved in our spectra. The different width of the splitting is probably due to differences in relaxation energy for the various final states, which the one-electron DOS does not take into account. This double structure of the $\pi^{*}$ resonance is associated with two zero-slope points along the $\overline{M K}$ high-symmetry direction in the band structure of graphene [8]. Therefore, the peak at $283.7 \mathrm{eV}$ cannot be related to a zigzag edge effect of graphene, but must be attributed to the peculiar unoccupied DOS of a single layer of carbon atoms.

Another interesting aspect in the NEXAFS spectrum of graphene, as well as of FLG, is a feature located at about $288 \mathrm{eV}$ (Fig. 2), between the $\pi^{*}$ and $\sigma^{*}$ resonances. This feature can be clearly seen in the spectrum of Fig. 4, obtained from the graphene flake of sample S2, where a higher spatial integration was performed. Considering the high inertness of graphene flakes, possible contamination of $\mathrm{C}-\mathrm{H}$ species at surface, which would give $\sigma^{*}(\mathrm{C}-\mathrm{H})$ transitions located between the $\pi^{*}$ and $\sigma^{*}$ resonances [20], are not expected to make a significant contribution. This was also confirmed by Raman spectra taken on the same samples. The upper inset of Fig. 4, indeed, shows the Raman spectrum of sample S2 taken at $514 \mathrm{~nm}$ and centered on the sharp 2D band, confirming for this region the thickness of one layer [12] and showing no structure related to $\nu(\mathrm{C}-\mathrm{H})$ vibrations at about $2900 \mathrm{~cm}^{-1}$ [21]. In addition, extreme care was taken to avoid sample damage or beam induced heating during the NEXAFS experiment. In the lower inset of Fig. 4, a close-up of NEXAFS spectra extracted from ticker flakes of sample S2 is also reported.

The peak located at about $288 \mathrm{eV}$, clearly defined in the photoabsorption spectrum of single and few-layer graphene, can be ascribed to a graphene analog of the interlayer state of graphite. This state has been the subject of theoretical and experimental investigations in the past, as it changed the commonly accepted description of the graphite band structure. Indeed, beside $\sigma$ and $\pi$ bonding states,

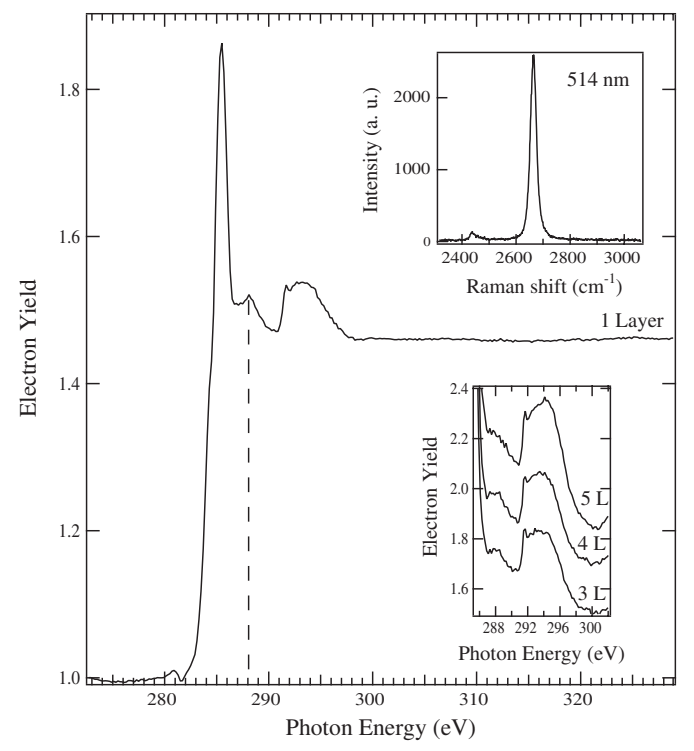

FIG. 4. C $K$-edge photoabsorption spectra of graphene taken from sample S2. Upper inset: the Raman spectrum of graphene at $514 \mathrm{~nm}$ showing the single component of the 2D peak. Lower inset: close-up of photoabsorption spectra extracted from sample S2. 
followed by $\pi^{*}$ and $\sigma^{*}$ antibonding states above the Fermi level, theory predicted an additional conduction band of three-dimensional character, namely, showing strong dispersion along the perpendicular direction [9]. This state was called interlayer state because its charge density is mainly confined between the basal planes, although it was believed to exist for a single layer of atoms as well. It helped to understand the electronic behavior of alkali-metal graphite intercalation compounds (AGICs) [9,22], demonstrating that no additional state arises from the alkali $s$ electrons, and that the free-electron band of AGICs preexists above the Fermi level in pure graphite. The interlayer state was first observed in HOPG by angle-resolved inverse photoemission [23] and NEXAFS [10]. Nevertheless, the possibility to detect this state by C $K$ TEY spectra of pure HOPG was questioned [24], and its location with respect to the Fermi level was also a source of controversy [25]. However, recent experiments and theoretical calculations performed on singlecrystal graphite [26] have clearly determined the position and dispersion of three-dimensional unoccupied bands of graphite, including the interlayer state. According to this investigation, the lower branch of the interlayer state exhibits almost dispersionless behavior at the $\Gamma$ point of the $\mathrm{BZ}$, at about $5 \mathrm{eV}$ from $E_{F}$. The present results clearly show a structure in the same energy region, similar to NEXAFS data on HOPG [10], and establish the existence of the interlayer state in graphene. These observations were confirmed also for sample S3, showing that graphene flakes deposited on $\mathrm{SiO}_{2}$ do behave, with respect to the electronic properties probed by a NEXAFS investigation, like suspended membranes.

In conclusion, laterally resolved absorption spectroscopy performed in UHV conditions by a PEEM has allowed us to establish the C $K$ edge NEXAFS spectra of graphene. The data exhibit characteristic spectral features, reflecting specific properties of the unoccupied DOS of single-layer graphene. A comparison of spectra of singlelayer, bilayer, and FLG samples illustrates the rapid evolution of the electronic states from those of a truly twodimensional systems, towards those of bulk graphite.

We are grateful to Professor E. Cazzanelli for useful discussions. Part of this work was performed at the Swiss Light Source, Paul Scherrer Institut, Switzerland. This research project has been supported by the European Commission under the 6th Framework Programme through the Key Action: Strengthening the European Research Area, Research Infrastructures. Contract No. R113-CT2004-506008.

*Corresponding author.

dpacile@fis.unical.it

[1] K. S. Novoselov, A. K. Geim, S. V. Morosov, D. Jiang, Y. Zhang, S. V. Dubonos, I. V. Grigorieva, and A. A. Firsov, Science 306, 666 (2004).
[2] K.S. Novoselov, A. K. Geim, S. V. Morozov, D. Jiang, M. I. Katsnelson, I. V. Grigorieva, S. V. Dubonos, and A. A. Firsov, Nature (London) 438, 197 (2005).

[3] Y. B. Zhang, Y. -W. Tan, H. L. Stormer, and P. Kim, Nature (London) 438, 201 (2005).

[4] C. Oshima and A. Nagashima, J. Phys. Condens. Matter 9, 1 (1997).

[5] I. Forbeaux, J.M. Themlin, and J. M. Debever, Phys. Rev. B 58, 16396 (1998).

[6] S. Y. Zhou, G.-H. Gweon, A. V. Fedorov, P. N. First, W. A. de Heer, D.-H. Lee, F. Guinea, A. H. Castro Neto, and A. Lanzara, Nature Mater. 6, 770 (2007).

[7] A. Bostwick, T. Ohta, T. Seyller, K. Horn, and E. Rotenberg, Nature Phys. 3, 36 (2007).

[8] S. B. Trickey, F. Müller-Plathe, G. H. F. Diercksen and J. C. Boettger, Phys. Rev. B 45, 4460 (1992).

[9] M. Posternak, A. Baldereschi, A. J. Freeman, E. Wimmer, and M. Weinert, Phys. Rev. Lett. 50, 761 (1983).

[10] D. A. Fischer, R. M. Wentzcovitch, R. G. Carr, A. Continenza, and A. J. Freeman, Phys. Rev. B 44, 1427 (1991).

[11] Z.H. Ni, H. M. Wang, J. Kasim, H. M. Fan, T. Yu, Y. H. Wu, Y. P. Feng, and Z. X. Shen, Nano Lett. 7, 2758 (2007).

[12] A. C. Ferrari, J. C. Meyer, V. Scardaci, C. Casiraghi, M. Lazzeri, F. Mauri, S. Piscanec, D. Jiang, K. S. Novoselov, S. Roth, and A K. Geim, Phys. Rev. Lett. 97, 187401 (2006).

[13] C. Quitmann, U. Flechsig, L. Patthey, T. Schmidt, G. Ingold, M. Howells, M. Janousch, and R. Abela, Surf. Sci. 480, 173 (2001).

[14] R. A. Rosenberg, P. J. Love, and V. Rehn, Phys. Rev. B 33, 4034 (1986).

[15] B. Partoens and F. M. Peeters, Phys. Rev. B 74, 075404 (2006).

[16] J.E. Müller and J. W. Wilkins, Phys. Rev. B 29, 4331 (1984).

[17] X. Weng, P. Rez, and H. Ma, Phys. Rev. B 40, 4175 (1989).

[18] S. Entani, S. Ikeda, M. Kiguchi, K. Saiki, G. Yoshikawa, I. Nakai, H. Kondoh, and T. Ohta, Appl. Phys. Lett. 88, 153126 (2006).

[19] K. Nakada, M. Fujita, G. Dresselhaus, and M.S. Dresselhaus, Phys. Rev. B 54, 17954 (1996).

[20] J. Kikuma, K. Yoneyama, M. Nomura, T. Konishi, T. Hashimoto, R. Mitsumoto, Y. Ohuchi, and K. Seki, J. Electron Spectrosc. Relat. Phenom. 88, 919 (1998).

[21] H. B. Zhang, G. D. Lin, Z. H. Zhou, X. Dong, and T. Chen, Carbon 40, 2429 (2002).

[22] G. Csányi, P. B. Littlewood, A.H. Nevidomskyy, C. J. Pickard, and B.D. Simons, Nature Phys. 1, 42 (2005).

[23] Th. Fauster, F. J. Himpsel, J.E. Fischer, and E. W. Plummer, Phys. Rev. Lett. 51, 430 (1983).

[24] E.Z. Kurmaev, A. V. Galakhov, A. Moewes, S. Moehlecke, and Y. Kopelevich, Phys. Rev. B 66, 193402 (2002).

[25] P. E. Batson, Phys. Rev. B 48, 2608 (1993).

[26] V. N. Strocov, P. Blaha, H. I. Starnberg, M. Rohlfing, R. Claessen, J.-M. Debever, and J.-M. Themlin, Phys. Rev. B 61, 4994 (2000). 